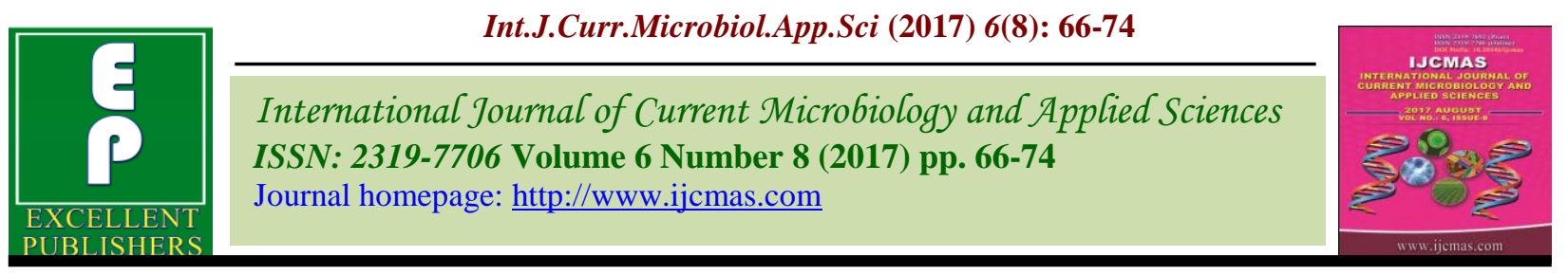

Original Research Article

https://doi.org/10.20546/ijcmas.2017.608.010

\title{
Study of Heterosis for Seed Yield and its Component Traits in Castor [Ricinus communis L.]
}

\author{
K.G. Kugashiya ${ }^{1 *}$, B.N. Patel ${ }^{2}$ and K.J. Vekariya ${ }^{1}$ \\ ${ }^{1}$ Department of Genetics and Plant Breeding, B. A. College of Agriculture, \\ Anand, 388 110, Gujarat, India \\ ${ }^{2}$ Agricultural Research Station, Anand Agricultural University, Sansoli, 387 130, Gujarat, India \\ *Corresponding author
}

\section{A B S T R A C T}

Keywords

Heterobeltiosis,

Standard

heterosis,

Seed yield.

Article Info

Accepted:

04 June 2017

Available Online:

10 August 2017
The phenomenon of heterosis has provided the most important genetic tools in improving yield of crop plants. Identification of specific parental combination capable of producing the highest level of heterotic effects in $F_{1}$ has immense value for commercial exploitation of heterosis. The experimental material comprised of 3 diverse pistillate lines, 13 inbred lines and their resultant 39 cross combinations and one standard check GCH 7 was evaluated in a randomized complete block design with three replications. Among 39 hybrids, 20 hybrids exhibited significant positive heterobeltiosis for seed yield, of these best three heterotic hybrids in order were SKP $84 \times$ ANDCI 8, SP 1 x SI 5 and SP 1 x DCS 100on the other hand three hybrids viz., SP1 x SI 5, SKP 84 x ANDCI 8 and SKP 84 x SI 5 exhibited significant positive standard heterosis over GCH 7.

\section{Introduction}

Castor (Ricinus communis L., $2 \mathrm{n}=2 \mathrm{X}=20$ ) resides one of the most important non-edible oilseed crops of India. Castor belongs to monospecific genus Ricinus of Euphorbiaceae family. Castor oil has great industrial utility along with it is used in pharmaceutical and cosmetics. Castor oil and cake are two major products obtained from castor seed. Castor oil is the source of sebacic acid, which is used in the manufacture of nylon and vinyl resins (Nagraj, 1996). In world, India ranks first in respect to area and production, contributing about 40 per cent of the global requirements. The other castor growing countries in the world were Brazil, China, Russia, Thailand, U.S.A. and a group of African countries. The area, production and productivity of castor in India during 2014-15 were 11.05 lakh ha, 17.33 lakh tonnes and $1568 \mathrm{~kg} / \mathrm{ha}$, respectively (Anonymous, 2015).

Nature and magnitude of heterosis is one of the important aspects for selection of the right parents for crosses and also help in identification of superior cross combinations that may produce desirable transgressive segregants in advanced generations. The choice of parents to be incorporated in 
hybridization programme is a crucial step for breeders, particularly if the aim is to improve the complex quantitative characters such as yield and its components. The use of parents of known superior genetic worth ensures much better success. It requires extensive and detailed genetic assessment of existing germplasm as well as newly developed promising genotype, which could be used in future breeding programme or could be directly released as a cultivar after thorough testing.

\section{Materials and Methods}

Experimental material of the study included 56 test entries comprising of three female lines (SP 1, VP 1 and SKP 84) and thirteen male parents (ANDCI 8, ANDCI 9DCS 100, DCS 9, JI 35, JI 384, JI 399, JI 402, JI 404, SI 4, SI 5, SI 6 and SI 9) and 39 resultant hybrids and one standard check hybrid GCH 7.

The crosses were made during kharif 2015-16 through Line $\mathrm{x}$ Tester mating design and evaluation was carried out during the year 2016-17 at Agricultural Research Station, Anand Agricultural University, Sansoli during kharif 2016-2017. Each entry was planted in a 6 meter long row with inter and intra row spacing of $120 \times 60 \mathrm{~cm}$. All the recommended agronomic and plant protection practices were uniformly applied throughout the crop growth period to raise a good crop.

The observations were recorded on five randomly selected plants for 12 characters in each replication for each genotype and the average value per plant was computed except for days to 50 per cent flowering and days to maturity of primary raceme. The observations of both these characters were recorded on population basis.

The replication wise mean values for all the characters were subjected to analysis of variance technique suggested by Snedecor and Cochran (1967) and reviewed by Panse and Sukhatme (1978) to determine significance differences among genotypes.

\section{Results and Discussion}

The analysis of variance was performed to test the differences among parents and hybrids for all the twelve characters and is presented in table 1 . The results revealed that the mean squares due to genotypes were significant for all the characters under study. The mean squares due to genotypes were further partitioned into parents, hybrids and parents $v s$. hybrids. The parents and hybrids differed significantly for all the characters. This revealed the existence of considerable genetic variability among the parents and hybrids for all the characters under study.

The analysis of variance further revealed that hybrids differed highly significantly for all the characters. The mean squares due to parents $v s$. hybrids were significant for all the characters, which indicated that the performance of parents was different from that of hybrids, thereby supporting the possibility of heterotic effects for all the traits. The mean squares due to check vs. hybrids were significant for all the traits except plant height upto primary raceme.

Seed yield is the economic important trait and breeders attempt to evolve varieties/hybrids with high seed yield. The results revealed that heterosis over better parent ranged from 18.68 (VP 1 x JI 404) to 56.21 (SKP 84 x ANDCI 8) percent; while, standard heterosis over GCH 7 varied from -49.69 (VP 1 x JI 404) and 23.77 (SP 1 x SI 5) percent. As many as 20 hybrids registered significant positive heterobeltiosis. Only three hybrids viz., SP 1 x SI 5 (23.77\%), SKP 84 x ANDCI $8(19.74 \%)$ and SKP 84 x SI $5(17.71 \%)$ recorded significant and positive standard 
heterosis over $\mathrm{GCH}$ 7. As observed in the present investigation, several workers have also reported the presence of considerable degree of heterosis for seed yield in castor
(Chaudhari and Patel, 2014; Chaudhari, 2007; Dave, 2015; Patel et al., 2010; Patel, 2013, 2014; Sasidharan, 2005; Thakker, 2002).

Table.1 Analysis of variance for yield and its components (Mean sum of squares)

\begin{tabular}{|c|c|c|c|c|c|c|c|}
\hline Source & d. f. & DF & NN & PH & DM & LP & NCP \\
\hline Replication & 2 & 3.74 & 2.25 & 138.5 & 7.13 & 21.07 & 60.57 \\
\hline Genotypes & 55 & $73.57 * *$ & $9.01 * *$ & $666.34 * *$ & $226.12 * *$ & $290.49 * *$ & $476.68 * *$ \\
\hline Parents & 15 & $85.7 * *$ & $8.36 * *$ & $228.51 * *$ & $172.12 * *$ & $98.81 * *$ & $238.65^{* *}$ \\
\hline Females & 2 & $165.44 * *$ & $18.9 * *$ & 140.65 & $240.33^{* *}$ & $465.5^{* *}$ & $232.75^{* *}$ \\
\hline Males & 12 & $65.94 * *$ & $5.67 * *$ & 48.34 & $114.47 * *$ & 41.22 & $255.29 * *$ \\
\hline Females vs Males & 1 & $163.36^{* *}$ & $19.56 * *$ & $2566.27 * *$ & $727.5^{* *}$ & 56.62 & 50.73 \\
\hline Hybrids & 38 & $64.82^{* *}$ & $8.47 * *$ & $539.52 * *$ & $196.01 * *$ & $300 * *$ & $539.62 * *$ \\
\hline Parents vs Hybrids & 1 & $149.6^{* *}$ & $10.41 * *$ & $12446.42 * *$ & $2191.4 * *$ & $25035.5 * *$ & $1531.34 * *$ \\
\hline Check vs Hybrids & 1 & $173.46^{* *}$ & $41.29 * *$ & 48.13 & $112.62 * *$ & $398.59 * *$ & $444.68 * *$ \\
\hline Error & 110 & 3.11 & 0.93 & 66.99 & 9.63 & 32.8 & 37.76 \\
\hline Source & d. f. & NEB & TNB & SY & TW & SH & OC \\
\hline Replication & 2 & 1.29 & 1.69 & 98 & 0.73 & 6.78 & 1.16 \\
\hline Genotypes & 55 & $12.96^{* *}$ & $11.61 * *$ & $5296.67 * *$ & $23.23 * *$ & $34.4 * *$ & $15.35 * *$ \\
\hline Parents & 15 & $12.22 * *$ & $11.53 * *$ & $1187.86^{* * *}$ & $17.29 * *$ & $17.54 * *$ & $15.66 * *$ \\
\hline Females & 2 & $25.41^{* *}$ & $26.01 * *$ & 346.57 & $28.18 * *$ & 3.07 & 0.3 \\
\hline Males & 12 & $6.23 * *$ & $8.35 * *$ & $1218.86^{* * *}$ & $16.91 * *$ & $21.26^{* *}$ & $17.75 * *$ \\
\hline Females vs Males & 1 & $57.68 * *$ & $20.77 * *$ & $2498.42 *$ & 0.14 & 1.85 & $21.36^{* *}$ \\
\hline Hybrids & 38 & $13.07 * *$ & $10.93 * *$ & $5705.31 * *$ & $25.36 * *$ & $34.65^{* *}$ & $14.85 * *$ \\
\hline Parents vs Hybrids & 1 & $6.82 *$ & $14.82 * *$ & $44152.75 * *$ & $8.18 *$ & $260.37 * *$ & $16.53 * *$ \\
\hline Check vs Hybrids & 1 & $23.87 * *$ & $31.18^{* *}$ & $8775.24 * *$ & $42.77 * *$ & $33.75^{* *}$ & $31.96^{* *}$ \\
\hline Error & 110 & 1.16 & 1.92 & 441.76 & 2.02 & 4.81 & 0.49 \\
\hline
\end{tabular}

Table.2 Promising hybrids for seed yield per plant with heterosisover standard check hybrid $(\mathrm{GCH} 7)$ and better parent (BP), their sca effects and component characters showing significant desired standard heterosis in castor

\begin{tabular}{|c|c|c|c|c|c|c|}
\hline \multirow{2}{*}{$\begin{array}{l}\text { Sr. } \\
\text { No }\end{array}$} & \multirow{2}{*}{ Hybrids } & \multirow{2}{*}{$\mathbf{S Y}(\mathbf{g})$} & \multicolumn{2}{|c|}{ Heterosis over } & \multirow{2}{*}{ sca effects } & \multirow{2}{*}{$\begin{array}{l}\text { Significant and desirable heterosis } \\
\text { over GCH } 7 \text { for component traits }\end{array}$} \\
\hline & & & BP & SC & & \\
\hline 1 & SP 1 x SI 5 & 315.22 & $47.26 * *$ & $23.77 * *$ & 10.72 & $\begin{array}{l}\text { DF, NN, PH, DM, LP, NCP, NEB, } \\
\text { TNB, TW and SH }\end{array}$ \\
\hline 2 & $\begin{array}{l}\text { SKP } 84 \times \text { ANDCI } \\
8\end{array}$ & 304.96 & $56.21 * *$ & $19.74 * *$ & $49.72 * *$ & $\mathrm{DF}, \mathrm{DM}, \mathrm{LP}, \mathrm{NCP}, \mathrm{TW}$ and $\mathrm{SH}$ \\
\hline 3 & SKP 84 x SI 5 & 299.79 & $40.06 * *$ & $17.71 * *$ & -12.8 & LP, NCP, TW and SH \\
\hline \multicolumn{2}{|c|}{ S. E \pm} & 12.13 & 17.30 & 17.30 & 8.31 & \\
\hline \multicolumn{7}{|c|}{$\begin{array}{l}\mathrm{DF}=\text { Days to } 50 \text { per cent flowering, NN = Number of nodes upto primary raceme, } \mathrm{PH}=\text { Plant height upto primary raceme }(\mathrm{cm}) \text {, } \\
\mathrm{DM}=\text { Days to maturity of primary raceme, } \mathrm{LP}=\text { Length of primary raceme }(\mathrm{cm}), \mathrm{NCP}=\text { Number of capsules on primary } \\
\text { raceme, NEB = Number of effective branches per plant, TNB = Total number of branches per plant, SY = Seed yield per plant } \\
(\mathrm{g}), \mathrm{TW}=100 \text { seed weight }(\mathrm{g})(\text { Test weight }), \mathrm{SH}=\text { Shelling out turn }(\%) \text { and } \mathrm{OC}=\text { Oil content }(\%)\end{array}$} \\
\hline
\end{tabular}


Table.3 Estimates of heterobeltiosis and standard heterosis for days to 50 per cent flowering, Number of nodes upto primary raceme and plant height upto primary raceme

\begin{tabular}{|c|c|c|c|c|c|c|c|}
\hline \multirow{2}{*}{ Sr. No. } & \multirow{2}{*}{ Hybrids } & \multicolumn{2}{|c|}{ DF } & \multicolumn{2}{|c|}{$\mathbf{N N}$} & \multicolumn{2}{|c|}{ PH } \\
\hline & & BP & SH & BP & SH & BP & SH \\
\hline 1 & SP $1 \times$ ANDCI 8 & 0.00 & $-9.84 * *$ & -0.74 & $-16.67 * *$ & $27.25^{*}$ & $-24.14 * *$ \\
\hline 2 & SP $1 \times$ ANDCI 9 & $-10.06 * *$ & $-16.94 * *$ & -4.78 & $-20.06 * *$ & $50.13 * *$ & -10.50 \\
\hline 3 & SP $1 \times$ DCS 100 & $-7.10 * *$ & $-14.21 * *$ & -5.51 & $-20.68 * *$ & $46.92 * *$ & -12.41 \\
\hline 4 & SP 1 × DCS 9 & -2.37 & $-9.84 * *$ & -6.25 & $-21.30 * *$ & 12.21 & $-33.10^{* * *}$ \\
\hline 5 & SP 1 × JI 35 & $-5.36^{*}$ & $-13.11 * *$ & -5.15 & $-20.37 * *$ & $50.90 * *$ & -10.04 \\
\hline 6 & SP $1 \times$ JI 384 & $-8.28 * *$ & $-15.30 * *$ & $-14.71 * *$ & $-28.40 * *$ & $51.41 * *$ & -9.73 \\
\hline 7 & SP $1 \times$ JI 399 & 1.18 & $-6.56^{* * *}$ & 1.10 & $-15.12 * *$ & $53.86 * *$ & -8.28 \\
\hline 8 & SP $1 \times$ JI 402 & -1.18 & $-8.74 * *$ & -1.10 & $-16.98 * *$ & $57.71 * *$ & -5.98 \\
\hline 9 & SP $1 \times$ JI 404 & -3.85 & $-31.69 * *$ & 0.00 & $-32.72 * *$ & 0.13 & $-40.31 * *$ \\
\hline 10 & SP $1 \times$ SI 4 & 2.40 & $-6.56 * *$ & -0.37 & $-16.36^{* *}$ & $52.06 * *$ & -9.35 \\
\hline 11 & SP $1 \times$ SI 5 & 1.18 & $-6.56^{* *}$ & 1.84 & $-14.51 * *$ & $33.29 *$ & $-20.54 * *$ \\
\hline 12 & SP $1 \times$ SI 6 & $-11.54 * *$ & $-24.59 * *$ & $-15.44 * *$ & $-29.01 * *$ & $35.60 * *$ & $-19.16^{*}$ \\
\hline 13 & SP $1 \times$ SI 9 & -2.96 & $-10.38 * *$ & $-9.56^{*}$ & $-24.07 * *$ & $55.53 * *$ & -7.28 \\
\hline 14 & VP 1 × ANDCI 8 & $31.01 * *$ & $-7.65 * *$ & $36.62 * *$ & $-10.19 * *$ & $107.53^{* *}$ & -2.84 \\
\hline 15 & VP 1× ANDCI 9 & $25.58 * *$ & $-11.48 * *$ & $24.41 * *$ & $-18.21 * *$ & $111.13^{* *}$ & -1.15 \\
\hline 16 & VP $1 \times$ DCS 100 & $12.40 * *$ & $-20.77 * *$ & $15.49 * *$ & $-24.07 * *$ & $113.26^{* *}$ & 0.61 \\
\hline 17 & VP 1× DCS 9 & $18.60 * *$ & $-16.39 * *$ & $25.82 * *$ & $-17.28 * *$ & $113.58 * *$ & 0.00 \\
\hline 18 & VP 1× JI 35 & $11.63 * *$ & $-21.31 * *$ & $18.31 * *$ & $-22.22 * *$ & $108.35^{* *}$ & -2.45 \\
\hline 19 & VP 1× JI 384 & -0.78 & $-30.05 * *$ & 4.23 & $-31.48 * *$ & 31.26 & $-38.54 * *$ \\
\hline 20 & VP 1× JI 399 & $13.95 * *$ & $-19.67 * *$ & $23.94 * *$ & $-18.52 * *$ & $91.49 * *$ & -10.34 \\
\hline 21 & VP 1× JI 402 & $22.48 * *$ & $-13.66 * *$ & $21.60 * *$ & $-20.06 * *$ & $91.98 * *$ & -10.11 \\
\hline 22 & VP 1× JI 404 & 5.43 & $-25.68 * *$ & $15.02 * *$ & $-24.38 * *$ & $87.23 * *$ & -12.34 \\
\hline 23 & VP 1× SI 4 & $24.81 * *$ & $-12.02 * *$ & $21.60 * *$ & $-20.06 * *$ & $88.05 * *$ & -11.95 \\
\hline 24 & VP $1 \times$ SI 5 & $31.78 * *$ & $-7.10 * *$ & $24.88 * *$ & $-17.90 * *$ & $98.36 * *$ & -7.13 \\
\hline 25 & VP 1× SI 6 & $26.36 * *$ & $-10.93 * *$ & $15.02 * *$ & $-24.38 * *$ & $102.13 * *$ & -5.36 \\
\hline 26 & VP 1× SI 9 & 3.88 & $-26.78 * *$ & 9.39 & $-28.09 * *$ & $108.84 * *$ & -2.22 \\
\hline 27 & SKP 84× ANDCI 8 & 3.64 & $-6.56^{* *}$ & $8.63 *$ & -6.79 & $86.34 * *$ & 13.95 \\
\hline 28 & SKP 84× ANDCI 9 & $6.02 *$ & -3.83 & 7.07 & -6.48 & $88.97 * *$ & $15.56^{*}$ \\
\hline 29 & SKP 84× DCS 100 & 2.41 & $-7.10^{* *}$ & 2.12 & $-10.80 * *$ & $54.76 * *$ & -5.36 \\
\hline 30 & SKP 84× DCS 9 & 3.61 & $-6.01 *$ & 4.24 & $-8.95^{*}$ & $64.91 * *$ & 0.84 \\
\hline 31 & SKP 84× JI 35 & -2.41 & $-11.48 * *$ & 2.94 & $-13.58 * *$ & $98.25 * *$ & $21.23 * *$ \\
\hline 32 & SKP 84× JI 384 & -2.41 & $-11.48 * *$ & $-8.48^{*}$ & $-20.06 * *$ & $80.70 * *$ & 10.50 \\
\hline 33 & SKP 84× JI 399 & 3.61 & $-6.01 *$ & $12.06 * *$ & -2.47 & $84.96 * *$ & 13.10 \\
\hline 34 & SKP 84× JI 402 & 4.22 & $-5.46^{*}$ & $8.83 *$ & -4.94 & $62.78 * *$ & -0.46 \\
\hline 35 & SKP 84× JI 404 & $16.15^{* *}$ & $-17.49 * *$ & $18.81 * *$ & $-20.06 * *$ & $62.16^{* *}$ & -0.84 \\
\hline 36 & SKP 84× SI 4 & 0.60 & $-8.74 * *$ & 2.52 & $-12.04 * *$ & $102.76^{* *}$ & $23.98 * *$ \\
\hline 37 & SKP 84× SI 5 & $8.43 * *$ & -1.64 & $10.60 *$ & -3.40 & $117.42 * *$ & $32.95 * *$ \\
\hline 38 & SKP 84× SI 6 & $10.90 * *$ & $-5.46^{*}$ & $9.16^{*}$ & $-8.02 *$ & $58.77 * *$ & -2.15 \\
\hline 39 & SKP 84× SI 9 & $6.63^{*}$ & -3.28 & 5.65 & $-7.72 *$ & $81.58 * *$ & 11.03 \\
\hline \multirow[t]{2}{*}{ Range } & Min & -11.54 & -31.69 & -15.44 & -32.72 & 0.13 & -40.31 \\
\hline & Max & 31.78 & -1.64 & 36.62 & -2.47 & 117.42 & 32.95 \\
\hline & S. E. \pm & 1.45 & 1.45 & 0.79 & 0.79 & 6.73 & 6.73 \\
\hline \multicolumn{2}{|c|}{ No of significant crosses } & 20 & 36 & 21 & 34 & 36 & 10 \\
\hline \multirow{2}{*}{\multicolumn{2}{|c|}{$\begin{array}{c}\text { Positive } \\
\text { Negative }\end{array}$}} & 15 & 0 & 17 & 0 & 36 & 4 \\
\hline & & 5 & 36 & 4 & 34 & 0 & 6 \\
\hline
\end{tabular}


Table.4 Estimates of heterobeltiosis and standard heterosis for day's maturity of primary raceme, Length of primary raceme and number of capsules on primary raceme

\begin{tabular}{|c|c|c|c|c|c|c|c|}
\hline \multirow{2}{*}{ Sr. No. } & \multirow{2}{*}{ Hybrids } & \multicolumn{2}{|c|}{ DM } & \multicolumn{2}{|c|}{ LP } & \multicolumn{2}{|c|}{ NCP } \\
\hline & & BP & SH & BP & SH & BP & SH \\
\hline $\mathbf{1}$ & SP $1 \times$ ANDCI 8 & $18.81 * *$ & 1.41 & 2.44 & $\begin{array}{l}-10.63 \\
\end{array}$ & 5.76 & $-12.04 *$ \\
\hline 2 & SP $1 \times$ ANDCI 9 & $13.53 * *$ & -3.10 & $-14.53^{*}$ & $-25.43 * *$ & -2.61 & $-19.00 * *$ \\
\hline 3 & SP $1 \times$ DCS 100 & $14.52 * *$ & -2.25 & -0.72 & $-13.39 *$ & 6.38 & $-11.52^{*}$ \\
\hline 4 & SP $1 \times$ DCS 9 & $18.48^{* *}$ & 1.13 & -6.95 & $-18.82 * *$ & 4.77 & $-12.86^{*}$ \\
\hline 5 & SP $1 \times$ JI 35 & $18.81 * *$ & 1.41 & 0.45 & $-12.36^{*}$ & 1.44 & $-15.63 * *$ \\
\hline 6 & SP $1 \times$ JI 384 & $18.48^{* *}$ & 1.13 & -1.26 & $-13.86^{*}$ & 8.27 & -9.95 \\
\hline 7 & SP 1 × JI 399 & $20.79 * *$ & 3.10 & 3.79 & -9.45 & 5.94 & $-11.89^{*}$ \\
\hline 8 & SP $1 \times$ JI 402 & $5.94 *$ & $-9.58 * *$ & 1.99 & $-11.02 *$ & $17.90 * *$ & -1.94 \\
\hline 9 & SP $1 \times$ JI 404 & 2.95 & $-21.41 * *$ & $-12.82 *$ & $-23.94 * *$ & 1.53 & $-15.56 * *$ \\
\hline 10 & SP $1 \times$ SI 4 & $5.94 *$ & $-9.58^{* *}$ & $13.27 *$ & -1.18 & $11.36^{*}$ & 10.02 \\
\hline 11 & SP $1 \times$ SI 5 & $4.95^{*}$ & $-10.42 * *$ & $34.75^{* *}$ & $17.56^{* *}$ & $25.13 * *$ & $30.74 * *$ \\
\hline 12 & SP $1 \times$ SI 6 & -3.00 & $-18.03 * *$ & $-14.44 *$ & $-25.35 * *$ & -5.76 & $-21.62 * *$ \\
\hline 13 & SP $1 \times$ SI 9 & $7.26 * *$ & $-8.45^{* *}$ & $\begin{array}{l}-7.04 \\
\end{array}$ & $-18.90 * *$ & -1.98 & $-18.47 * *$ \\
\hline 14 & VP 1 × ANDCI 8 & $29.18 * *$ & $-6.48 * *$ & 12.43 & -7.40 & 10.21 & $-20.04 * *$ \\
\hline 15 & VP 1× ANDCI 9 & $32.30 * *$ & $-4.23 *$ & 6.08 & $-13.46^{*}$ & 9.54 & $-21.02 * *$ \\
\hline 16 & VP $1 \times$ DCS 100 & $17.90^{* *}$ & $-14.65 * *$ & 7.93 & $-12.13^{*}$ & 6.00 & $-15.41 * *$ \\
\hline 17 & VP 1× DCS 9 & $23.35 * *$ & $-10.70 * *$ & 14.39 & $-15.51 * *$ & 15.33 & $-17.28 * *$ \\
\hline 18 & VP 1× JI 35 & $16.73 * *$ & $-15.49 * *$ & $36.19^{* *}$ & 1.34 & $20.02 * *$ & -7.63 \\
\hline 19 & VP 1× JI 384 & 17.90 ** & $-14.65 * *$ & 11.01 & $-17.40 * *$ & 4.44 & $-22.51 * *$ \\
\hline 20 & VP 1× JI 399 & $35.02 * *$ & -2.25 & 13.23 & $-19.13 * *$ & 7.29 & $-16.31 * *$ \\
\hline 21 & VP 1× JI 402 & $38.91 * *$ & 0.56 & 2.87 & $-26.54 * *$ & -2.66 & $-23.41 * *$ \\
\hline 22 & VP 1× JI 404 & $14.01 * *$ & $-17.46 * *$ & -11.76 & $-35.04 * *$ & $-15.91 *$ & $-37.55^{* *}$ \\
\hline 23 & VP 1×SI 4 & $37.35^{* *}$ & -0.56 & 9.40 & $-11.10^{*}$ & $-13.85^{*}$ & $-14.88^{* *}$ \\
\hline 24 & VP 1× SI 5 & $32.68 * *$ & -3.94 & $19.71^{* *}$ & -1.02 & 7.37 & $12.19 *$ \\
\hline 25 & VP 1× SI 6 & $31.13 * *$ & $-5.07 *$ & 0.00 & $-26.06 * *$ & $\begin{array}{l}-6.79 \\
\end{array}$ & $-31.19 * *$ \\
\hline 26 & VP 1× SI 9 & $21.79 * *$ & $-11.83^{* *}$ & -8.11 & $-31.34 * *$ & -9.79 & $-33.13 * *$ \\
\hline 27 & SKP 84× ANDCI 8 & $8.55 * *$ & $-7.04 * *$ & $38.15^{* *}$ & $13.78^{*}$ & $43.88 * *$ & $16.01 * *$ \\
\hline 28 & SKP 84× ANDCI 9 & $10.53^{* *}$ & $-5.35^{*}$ & -1.83 & $-19.92 * *$ & 2.13 & $-17.65^{* *}$ \\
\hline 29 & SKP 84× DCS 100 & $15.13^{* * *}$ & -1.41 & 0.87 & $-17.87 * *$ & 2.23 & $-17.58 * *$ \\
\hline 30 & SKP 84× DCS 9 & $14.80^{* *}$ & -1.69 & $17.21 *$ & $-12.05^{*}$ & 12.62 & -9.20 \\
\hline 31 & SKP 84× JI 35 & $17.76^{* *}$ & 0.85 & $17.52 *$ & $-11.81^{*}$ & 12.34 & -9.42 \\
\hline 32 & SKP 84× JI 384 & $13.82^{* * *}$ & -2.54 & -0.84 & $-25.59 * *$ & -4.55 & $-23.04 * *$ \\
\hline 33 & SKP 84× JI 399 & $16.78^{* * *}$ & 0.00 & -3.04 & $-27.24 * *$ & -6.49 & $-24.61 * *$ \\
\hline 34 & SKP 84× JI 402 & $18.09^{* * *}$ & 1.13 & 0.63 & $-24.49 * *$ & -12.52 & $-29.47 * *$ \\
\hline 35 & SKP 84× JI 404 & $13.65^{* *}$ & $-13.24 * *$ & $16.58^{*}$ & $-12.52 *$ & -9.00 & $-26.63 * *$ \\
\hline 36 & SKP 84× SI 4 & $19.08 * *$ & 1.97 & $22.48 * *$ & -0.47 & 10.45 & 9.12 \\
\hline 37 & SKP 84× SI 5 & $25.33^{* *}$ & $7.32 * *$ & $36.19 * *$ & $12.60 *$ & 9.45 & $14.36^{*}$ \\
\hline 38 & SKP 84× SI 6 & $18.00^{* * *}$ & -0.28 & 13.96 & $-14.49 * *$ & $-15.12^{*}$ & $-31.56 * *$ \\
\hline 39 & SKP 84× SI 9 & $13.49^{* * *}$ & -2.82 & 11.86 & $-16.06^{* *}$ & $-15.40^{*}$ & $-31.79 * *$ \\
\hline \multirow{2}{*}{ Range } & Min & -3.00 & -21.41 & -14.53 & -35.04 & -15.91 & -37.55 \\
\hline & Max & 38.91 & 7.32 & 38.15 & 17.56 & 43.88 & 30.74 \\
\hline & S. E. \pm & 2.55 & 2.55 & 4.71 & 4.71 & 5.05 & 5.05 \\
\hline \multicolumn{2}{|c|}{ No of significant crosses } & 37 & 19 & 13 & 32 & 9 & 32 \\
\hline \multicolumn{2}{|c|}{ Positive } & 37 & 1 & 10 & 3 & 5 & 4 \\
\hline & Negative & 0 & 18 & 3 & 29 & 4 & 28 \\
\hline
\end{tabular}


Table.5 Estimates of heterobeltiosis and standard heterosis for number of effective branches per Plant, total number of branches per plant and seed yield per plant

\begin{tabular}{|c|c|c|c|c|c|c|c|}
\hline \multirow{2}{*}{ Sr. No. } & \multirow{2}{*}{ Hybrids } & \multicolumn{2}{|c|}{ NEB } & \multicolumn{2}{|c|}{ TNB } & \multicolumn{2}{|c|}{ SY } \\
\hline & & BP & SH & BP & SH & BP & SH \\
\hline 1 & SP $1 \times$ ANDCI 8 & 0.00 & 0.95 & -5.45 & -11.64 & 12.95 & $-13.42 *$ \\
\hline 2 & SP $1 \times$ ANDCI 9 & $-13.68 *$ & $-12.86^{*}$ & -11.67 & $-17.45 * *$ & 13.69 & $-26.91 * *$ \\
\hline 3 & SP $1 \times$ DCS 100 & -8.49 & -7.62 & -8.17 & $-14.18 *$ & $43.13 * *$ & -12.07 \\
\hline 4 & SP 1 × DCS 9 & $-22.17 * *$ & $-21.43 * *$ & $-16.73^{2}$ & $-22.18 * *$ & $26.30 *$ & $-22.41 * *$ \\
\hline 5 & SP 1 × JI 35 & $-16.04 *$ & $-15.24 *$ & -5.06 & -11.27 & $20.55^{*}$ & $-21.63 * *$ \\
\hline 6 & SP 1 × JI 384 & $-28.77 * *$ & $-28.10 * *$ & $-20.62 * *$ & $-25.82 * *$ & 17.04 & $-28.10 * *$ \\
\hline 7 & SP 1 × JI 399 & $-27.83 * *$ & $-27.14 * *$ & -9.73 & $-15.64 *$ & 5.71 & $-27.81 * *$ \\
\hline 8 & SP $1 \times$ JI 402 & $-15.09 *$ & $-14.29 *$ & -3.89 & $\begin{array}{l}-10.18 \\
\end{array}$ & $22.69 *$ & $-23.25 * *$ \\
\hline 9 & SP $1 \times$ JI 404 & $-20.75 * *$ & $-20.00 * *$ & $-14.01 *$ & $-19.64 * *$ & $23.04 *$ & $-23.87 * *$ \\
\hline 10 & SP $1 \times$ SI 4 & -2.83 & -1.90 & 3.50 & -3.27 & $21.59 *$ & -12.41 \\
\hline 11 & SP $1 \times$ SI 5 & $20.28^{* *}$ & $21.43 * *$ & $24.43 * *$ & $18.55^{* *}$ & $47.26^{* *}$ & $23.77 * *$ \\
\hline 12 & SP $1 \times$ SI 6 & $-31.13 * *$ & $-30.48 * *$ & $-27.24 * *$ & $-32.00 * *$ & 5.41 & $-34.5 * *$ \\
\hline 13 & SP $1 \times$ SI 9 & $-28.30 * *$ & $-27.62 * *$ & -12.06 & $-17.82 * *$ & 5.60 & $-31.46 * *$ \\
\hline 14 & VP 1 × ANDCI 8 & $-19.25 * *$ & $-28.10^{* *}$ & -5.60 & $-20.36 * *$ & 2.97 & $-21.07 * *$ \\
\hline 15 & VP 1× ANDCI 9 & -5.84 & $-30.95 * *$ & -1.83 & $-22.18 * *$ & 18.05 & $-24.11 * *$ \\
\hline 16 & VP 1× DCS 100 & 3.92 & $-24.29 * *$ & -7.37 & $-26.91 * *$ & $25.65^{*}$ & $-27.05 * *$ \\
\hline 17 & VP 1× DCS 9 & 13.10 & $-21.90 * *$ & $24.19^{* *}$ & $-16.00 * *$ & $29.61 *$ & $-28.04 * *$ \\
\hline 18 & VP 1× JI 35 & $25.93 * *$ & -2.86 & $19.03 *$ & -2.18 & $33.81 * *$ & -13.02 \\
\hline 19 & VP 1× JI 384 & 4.61 & $-24.29 * *$ & 9.95 & $-15.64 *$ & 19.48 & $-29.60 * *$ \\
\hline 20 & VP 1× JI 399 & $-23.17 * *$ & $-40.00 * *$ & -12.56 & $-29.09 * *$ & -17.68 & $-43.78 * *$ \\
\hline 21 & VP 1× JI 402 & -11.72 & $-39.05 * *$ & 7.77 & $-24.36 * *$ & -10.46 & $-43.99 * *$ \\
\hline 22 & VP 1× JI 404 & $-26.90 * *$ & $-49.52 * *$ & -10.26 & $-36.36 * *$ & -18.68 & $-49.69 * *$ \\
\hline 23 & VP 1× SI 4 & 9.04 & $-13.81 *$ & 0.86 & $-14.91 *$ & $28.53 * *$ & -7.42 \\
\hline 24 & VP $1 \times$ SI 5 & 4.71 & -4.76 & -2.67 & -7.27 & $32.45^{* *}$ & 11.32 \\
\hline 25 & VP 1× SI 6 & -11.72 & $-39.05 * *$ & 5.91 & $-28.36 * *$ & -17.64 & $-48.83 * *$ \\
\hline 26 & VP 1× SI 9 & -6.21 & $-35.24 * *$ & 10.75 & $-25.09 * *$ & -17.61 & $-46.53 * *$ \\
\hline 27 & SKP 84× ANDCI 8 & -1.32 & 6.67 & -3.75 & -6.55 & $56.21 * *$ & $19.74 * *$ \\
\hline 28 & SKP 84× ANDCI 9 & $-26.87 * *$ & $-20.95 * *$ & $-15.73 *$ & $-18.18 * *$ & 8.52 & $-30.24 * *$ \\
\hline 29 & SKP 84× DCS 100 & $-23.79 * *$ & $-17.62 * *$ & -11.61 & $-14.18^{*}$ & $37.28^{* *}$ & $-17.20^{*}$ \\
\hline 30 & SKP 84× DCS 9 & $-24.67 * *$ & $-18.57 * *$ & $-15.36^{*}$ & $-17.82 * *$ & $34.54 * *$ & $-18.85 * *$ \\
\hline 31 & SKP 84× JI 35 & $-20.70 * *$ & $-14.29 *$ & $-14.98^{*}$ & $-17.45 * *$ & $41.6^{* *}$ & -7.96 \\
\hline 32 & SKP 84× JI 384 & $-37.00 * *$ & $-31.90 * *$ & $-22.47 * *$ & $-24.73 * *$ & $22.18^{*}$ & $-26.30 * *$ \\
\hline 33 & SKP 84× JI 399 & $-25.55 * *$ & $-19.52 * *$ & -11.61 & $-14.18^{*}$ & 7.94 & $-26.29 * *$ \\
\hline 34 & SKP 84× JI 402 & $-43.17 * *$ & $-38.57 * *$ & $-29.96 * *$ & $-32.00 * *$ & 12.68 & $-29.52 * *$ \\
\hline 35 & SKP 84× JI 404 & $-48.90 * *$ & $-44.76 * *$ & $-32.21 * *$ & $-34.18 * *$ & 10.73 & $-31.49 * *$ \\
\hline 36 & SKP 84× SI 4 & $-19.82 * *$ & $-13.33^{*}$ & -9.74 & $-12.36^{*}$ & $18.58 *$ & $-14.58^{*}$ \\
\hline 37 & SKP 84× SI 5 & -5.73 & 1.90 & -1.12 & -4.00 & $40.06^{* *}$ & $17.71 * *$ \\
\hline 38 & SKP 84× SI 6 & $-30.40 * *$ & $-24.76^{* * *}$ & $-18.35^{* *}$ & $-20.73 * *$ & $25.77 *$ & $-21.85 * *$ \\
\hline 39 & SKP 84× SI 9 & $-27.75 * *$ & $-21.90 * *$ & $-24.72 * *$ & $-26.91 * *$ & 14.01 & $-26.00 * *$ \\
\hline \multirow{2}{*}{ Range } & Min & -48.90 & -49.52 & -32.21 & -36.36 & -18.68 & -49.69 \\
\hline & Max & 25.93 & 21.43 & 24.43 & 18.55 & 56.21 & 23.77 \\
\hline & S. E. \pm & 0.88 & 0.88 & 1.13 & 1.13 & 17.30 & 17.30 \\
\hline \multicolumn{2}{|c|}{ No of significant crosses } & 25 & 32 & 15 & 31 & 20 & 33 \\
\hline \multirow{2}{*}{\multicolumn{2}{|c|}{$\begin{array}{c}\text { Positive } \\
\text { Negative }\end{array}$}} & 2 & 1 & 3 & 1 & 20 & 3 \\
\hline & & 23 & 31 & 12 & 30 & 0 & 30 \\
\hline
\end{tabular}


Table.6 Estimates of heterobeltiosis and standard heterosis for 100 seed weight, Shelling out turn and oil content

\begin{tabular}{|c|c|c|c|c|c|c|c|}
\hline \multirow{2}{*}{ Sr. No. } & \multirow{2}{*}{ Hybrids } & \multicolumn{2}{|c|}{ TW } & \multicolumn{2}{|c|}{ SH } & \multicolumn{2}{|c|}{ OC } \\
\hline & & BP & SH & BP & SH & BP & SH \\
\hline 1 & SP $1 \times$ ANDCI 8 & -1.51 & $-8.71 *$ & -3.63 & -3.13 & -0.18 & -1.55 \\
\hline 2 & SP $1 \times$ ANDCI 9 & $-11.37 * *$ & $-17.85 * *$ & 2.08 & $-7.77 * *$ & $-3.19 * *$ & $-5.61 * *$ \\
\hline 3 & SP $1 \times$ DCS 100 & -0.08 & $-7.39 *$ & -0.43 & $-9.73 * *$ & -0.15 & $-2.64 *$ \\
\hline 4 & SP 1 × DCS 9 & -4.41 & $-11.40 * *$ & 0.59 & $-10.26 * *$ & $-7.51 * *$ & $-9.82 * *$ \\
\hline 5 & SP 1 × JI 35 & -1.24 & $-8.46^{*}$ & 2.54 & -4.77 & -2.30 & $-4.74 * *$ \\
\hline 6 & SP 1 × JI 384 & $-10.93 * *$ & $-17.44 * *$ & 4.36 & $-6.91 *$ & -1.33 & $-3.79 * *$ \\
\hline 7 & SP $1 \times$ JI 399 & -0.85 & $-8.10^{*}$ & -2.14 & $-12.70 * *$ & $-12.17 * *$ & $-14.37 * *$ \\
\hline 8 & SP $1 \times$ JI 402 & $-7.07 *$ & $-10.60 * *$ & 0.84 & $-9.08 * *$ & -0.60 & $-3.08 * *$ \\
\hline 9 & SP $1 \times$ JI 404 & -4.01 & $-9.70^{* *}$ & $7.21 *$ & -3.50 & $-6.31 * *$ & $-8.65^{* *}$ \\
\hline 10 & SP $1 \times$ SI 4 & 7.01 & -0.81 & 3.55 & $-5.83 *$ & $-3.86^{* *}$ & $-6.26 * *$ \\
\hline 11 & SP $1 \times$ SI 5 & $7.82^{*}$ & $11.73 * *$ & $12.96^{* *}$ & $7.23^{*}$ & -0.87 & -1.61 \\
\hline 12 & SP $1 \times$ SI 6 & $-7.87 *$ & $-14.61 * *$ & -1.95 & $-12.53 * *$ & -0.93 & $-3.41 * *$ \\
\hline 13 & SP $1 \times$ SI 9 & $-13.24 * *$ & $-19.58 * *$ & -2.08 & $-12.65 * *$ & $-11.77 * *$ & $-13.98 * *$ \\
\hline 14 & VP 1 × ANDCI 8 & 6.10 & $-8.80^{* *}$ & -1.72 & -1.21 & -0.97 & $-2.33^{*}$ \\
\hline 15 & VP 1× ANDCI 9 & 5.45 & $-15.89 * *$ & $7.50 *$ & -2.07 & $-5.37 * *$ & $-8.37 * *$ \\
\hline 16 & VP $1 \times$ DCS 100 & -4.50 & $-19.47 * *$ & 2.27 & $-6.85^{*}$ & $-2.81 *$ & $-5.89 * *$ \\
\hline 17 & VP 1× DCS 9 & -3.26 & $-19.71 * *$ & 3.17 & $-6.02 *$ & $-7.03^{* *}$ & $-9.99 * *$ \\
\hline 18 & VP 1× JI 35 & 6.39 & -5.34 & -4.33 & $-11.16 * *$ & -1.65 & $-4.22 * *$ \\
\hline 19 & VP 1× JI 384 & -6.25 & $-18.29 * *$ & 3.11 & $-6.08^{*}$ & $-10.43 * *$ & $-13.28 * *$ \\
\hline 20 & VP 1× JI 399 & $8.99 *$ & $-8.71 *$ & -0.19 & $-9.08 * *$ & $-5.01 * *$ & $-8.03 * *$ \\
\hline 21 & VP 1× JI 402 & $-11.46 * *$ & $-14.82 * *$ & $8.07 *$ & -1.56 & -2.18 & $-5.28 * *$ \\
\hline 22 & VP 1× JI 404 & $-21.79 * *$ & $-26.42 * *$ & -5.00 & $-13.47 * *$ & $-5.29 * *$ & $-8.30 * *$ \\
\hline 23 & VP 1× SI 4 & 4.11 & $-10.75 * *$ & $9.72 * *$ & -0.06 & $-7.72 * *$ & $-10.65 * *$ \\
\hline 24 & VP $1 \times$ SI 5 & $-11.31 * *$ & $-8.10^{*}$ & $9.02 * *$ & 3.48 & -1.09 & -1.82 \\
\hline 25 & VP 1× SI 6 & -4.97 & $-25.58 * *$ & -2.18 & $-10.89 * *$ & -1.86 & $-4.50 * *$ \\
\hline 26 & VP 1× SI 9 & $-12.08 * *$ & $-24.06 * *$ & -3.83 & $-12.40 * *$ & $-15.07 * *$ & $-17.77 * *$ \\
\hline 27 & SKP 84× ANDCI 8 & $18.10^{* *}$ & $8.39 *$ & $6.18^{*}$ & $6.72 *$ & 0.59 & -0.79 \\
\hline 28 & SKP 84× ANDCI 9 & $-11.48 * *$ & $-18.76 * *$ & 1.45 & $-8.34 * *$ & -2.29 & $-4.01 * *$ \\
\hline 29 & SKP 84× DCS 100 & -2.10 & $-10.15 * *$ & 5.20 & -4.63 & $-2.72 *$ & $-4.43 * *$ \\
\hline 30 & SKP 84× DCS 9 & -1.43 & $-9.54 * *$ & $6.99 *$ & $-5.94 *$ & $-13.86 * *$ & $-15.38 * *$ \\
\hline 31 & SKP 84× JI 35 & 0.36 & $-7.89 *$ & 5.31 & -2.20 & -1.81 & $-3.54 * *$ \\
\hline 32 & SKP 84× JI 384 & 3.51 & -5.00 & $6.47^{*}$ & -5.61 & $-8.63 * *$ & $-10.24 * *$ \\
\hline 33 & SKP 84× JI 399 & -1.63 & $-9.72 * *$ & -1.51 & $-13.41 * *$ & $-9.07 * *$ & $-10.67 * *$ \\
\hline 34 & SKP 84× JI 402 & $-7.20 *$ & $-10.73 * *$ & $9.31 * *$ & -1.54 & $-3.32 * *$ & $-5.02 * *$ \\
\hline 35 & SKP 84× JI 404 & -6.79 & $-12.31 * *$ & $8.28 * *$ & -2.55 & -1.54 & $-3.28 * *$ \\
\hline 36 & SKP 84× SI 4 & 5.83 & -2.87 & $7.63 *$ & -2.12 & $-2.39 *$ & $-4.11 * *$ \\
\hline 37 & SKP 84× SI 5 & 3.67 & $7.43 *$ & $12.10^{* *}$ & $6.41 *$ & -1.52 & -2.25 \\
\hline 38 & SKP 84× SI 6 & $-12.82 * *$ & $-19.99 * *$ & 6.20 & $-6.64 *$ & -1.29 & $-3.03 * *$ \\
\hline 39 & SKP 84× SI 9 & $-7.91 *$ & $-15.48 * *$ & $8.45^{*}$ & -4.65 & $-13.78 * *$ & $-15.30 * *$ \\
\hline \multirow{2}{*}{ Range } & Min & -21.79 & -26.42 & -5.00 & -13.47 & -15.07 & -17.77 \\
\hline & Max & 18.10 & 11.73 & 12.96 & 7.23 & 0.59 & -0.79 \\
\hline & S. E. \pm & 1.16 & 1.16 & 1.79 & 1.79 & 0.57 & 0.57 \\
\hline \multicolumn{2}{|c|}{ No of significant crosses } & 16 & 35 & 14 & 24 & 21 & 34 \\
\hline & Positive & 3 & 3 & 14 & 3 & 0 & 0 \\
\hline & Negative & 13 & 32 & 0 & 21 & 21 & 34 \\
\hline
\end{tabular}


Whereas for days to 50 per cent flowering and days to maturity of primary raceme hybrid SP 1 x JI 404 found most promising.

Comparison of top three promising crosses on the basis of per se performance for seed yield per plant along with sca effect and percent heterosis over better parent and standard check GCH 7 in different traits (Table 2). On the basis of per se performance and heterosis the hybrids SP 1 x SI 5, SKP 84 x ANDCI 8 and SKP $84 \times$ SI 5 were found the most promising and may be exploited in further breeding programme. Their superiority seems to have resulted from higher values of their yield contributing characters like length of primary raceme, number of capsules on primary raceme, number of effective branches per plant and 100-seed weight.

It can be concluded among 39 hybrids tested, three hybrids viz., SP 1 x SI 5, SKP 84 x ANDCI 8 and SKP 84 x SI 5 recorded significant and positive heterosis over GCH 7 for seed yield per plant (Tables 3-6). These hybrids also depicted significant and positive heterosis over their respective better parent. The high heterotic response in these hybrids resulted due to positive heterosis for yield contributing characters like length of primary raceme, number of capsules on primary raceme, effective number of branches per plant and 100 seed weight. The heterotic effect for seed yield per plant could be outcome of direct effect of these attributes, and could be outcome of indirect effects of other yield contributing attributes like number of effective branches per plant and number of capsules per plant. Therefore, heterotic effects for seed yield per plant could be a result of combinational heterosis.

\section{References}

Anonymous (2015). Area, production and yield per hectare of castor crop in
India. (Director's Report, IIOR, Hyderabad).

Chaudhari G. and Patel B. N. (2014). Heterosis and combining ability analysis for oil yield and its components in castor (Ricinus communis L.). Trends in Bioscience, 7(22), 3757-3760.

Chaudhari Y. V. (2007). Genetic studies in castor (Ricinus communis L.). Unpublished M. Sc. (Agri.) thesis submitted to Sardarkrushinagar Dantiwada Agricultural University, Sardarkrushinagar.

Dave P. B. (2015). Genetic architecture of seed yield and its component characters over environments in castor. Unpublished $\mathrm{Ph}$. D. thesis submitted to Anand Agricultural University, Anand (Gujarat).

Nagraj G. (1996). Composition and quality of oilseeds. In: Genetic Improvement of Oilseed Crops (Jafar N., Farook, S.A. and Khan, I.A. Ukaaz Ed.)Publications, Hyderabad (A.P.), pp. 265- 313.

Panse V. G. and Sukhatme P. V. (1978). Statistical methods for agricultural workers. ICAR, New Delhi.

Patel A. R., Patel B. N., Doshi J. S., Patel J. B., and Patel J. A. (2010). Heterosis for seed yield and its components over environments in castor (Ricinus communis L.). Journals of Oilseeds Research, 27, 53-57.

Patel K. P. (2013). Studies on heterosis, combining ability and gene action through diallel crosses in castor (Ricinus communis L.). Unpublished M. Sc. (Agri.) thesis submitted to Anand Agricultural University, Anand (Gujarat).

Patel K. R. (2004). Genetics of yield, yield components and wilt resistance in castor (Ricinus communis L.). Unpublished Ph. D. thesis submitted 
to Sardarkrushinagar Dantiwada Agricultural Sardarkrushinagar.

Sasidharan N. (2005). Genetic analysis for yield and quality in castor (Ricinus communis L.) under diverse environments. Unpublished $\mathrm{Ph}$. D. thesis submitted to Anand Agricultural University, Anand (Gujarat).
Snedecor G.W. and Cochran W.G. (1967). Statistical methods. $6^{\text {th }}$ ed. Ames: Iowa State University Press.

Thakker D. A. (2002). Heterosis and combining ability studies in castor (Ricinus communis L.). Unpublished $\mathrm{Ph}$. D. thesis submitted to Gujarat Agricultural University, Sardarkrushinagar.

\section{How to cite this article:}

Kugashiya, K.G., B.N. Patel and Vekariya, K.J. 2017. Study of Heterosis for Seed Yield and its Component Traits in Castor (Ricinus communis L.). Int.J.Curr.Microbiol.App.Sci. 6(8): 66-74. doi: https://doi.org/10.20546/ijcmas.2017.608.010 